proteins in brain, liver, muscle and knee joint. TG induced a significant expansion in the growth plate height and in the hypertropic zone height, in comparison to untreated mice, without a significant modification of body weight.

Conclusions TG induced the expression of chondrogenic markers in ATDC5 cells, and increased chondrogenic differentiation in vivo. Our results show that o-GlcNAc glycosylation has chondromodulating activity.

\section{O-GLCNAC PROTEIN MODIFICATION STIMULATES CHONDROGENESIS IN VITRO AND CHONDROCYTE HYPERTROPHY IN MOUSE}

Jessica Andrés-Bergós, Lidia Tardío, Amanda Villalvilla, Rodolfo Gómez, Gabriel Herrero-Beaumont and Raquel Largo Joint and Bone Research Unit. IIS-Fundación Jiménez Díaz, Universidad Autónoma Madrid. Spain

10.1136/annrheumdis-2011-201237.24

Background and objectives Chondrocyte differentiation that allows endocondral ossification sequentially includes cell proliferation, extracellular matrix synthesis, cellular hypertrophy, matrix mineralisation, vascular invasion and eventually apoptosis, allowing cartilage remodeling into bone. Most of these processes have been also associated to the development of osteoarthritis. Insulin ameliorate impaired bone growth and impaired bone healing both in vitro and in vivo, and is able to induce chondrocyte hypertrophy and growth plate chondrogenesis, although the specific molecular mechanisms are mostly unknown. Our aim was to investigate whether insulin-induced chondrocyte hypertrophy occurs through a modification in the amount of O-GlcNAc glycosylated proteins and in the expression of the key enzymes of this pathway: O-GlcNAc-transferase (OGT) and O-GlcNAcase (OGA). Furthermore, the authors studied if O-GlcNAc-accumulation per se was able to induce prehypertrophic chondrocyte differentiation both in vitro and in vivo.

Methods Prechondrogenic ATDC5 cells were cultured in 5\% FBS DMEM/F12. Insulin-induced differentiation was studied for 24 days. The accumulation of O-GlcNAcylated proteins was induced employing the OGA inhibitor Thiamet-G (TG). The gene expression of the differentiation markers collagen $\mathrm{X}$ (ColX), PTH receptor (PTHR), Indian hedgehog protein (IHH), Runx-2 and alkaline phosphatase (AP) was studied by real time-PCR. O-GlcNAcylation, OGT, OGA, extracellular signal-regulated kinase (ERK), c-Jun $\mathrm{N}$-terminal kinase (JNK) and p38 were studied by western-blot. The effect of the in vivo accumulation of O-GlcNAc proteins in the tibial growth plate was studied in 23-day old C57/bl mice that received $20 \mathrm{mg} / \mathrm{kg}$ TG during 15 days.

Results Insulin-induced differentiation paralleled with a gradual increase in the accumulation of O-GlcNacylated proteins that was appreciable since day 3 , and with an increase in the expression of OGT and OGA. These increases occurred previously to any modification in the differentiation markers. O-GlcNAc accumulation per se induced by TG, in the absence of insulin, induced ATDC5 differentiation measured by the increase in the expression of differentiation markers. TG cells also induced an increase in the activity of MMP-1,-2 and -9, similar to that evoked by insulin. TG activated pERK, p-JNK and p-p38 in a similar extent to insulin. The administration of TG to $\mathrm{C} 57 / \mathrm{bl}$ mice induced an accumulation of O-GlcNAcylated 